\title{
Probing Gluon Saturation through Dihadron Correlations at an Electron-Ion Collider
}

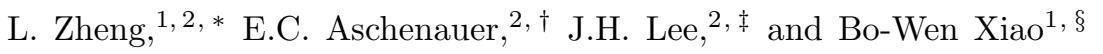 \\ ${ }^{1}$ Key Laboratory of Quark and Lepton Physics (MOE) and \\ Institute of Particle Physics, \\ Central China Normal University, \\ Wuhan 430079, China \\ ${ }^{2}$ Physics Department, Brookhaven National Laboratory, \\ Upton, NY 11973, U.S.A.
}

(Dated: April 8, 2014)

\begin{abstract}
Two-particle azimuthal angle correlations have been proposed to be one of the most direct and sensitive probes to access the underlying gluon dynamics involved in hard scatterings. In anticipation of an Electron-Ion Collider (EIC), detailed studies of dihadron correlation measurements in electronproton and electron-ion collisions at an EIC have been performed. The impact of such measurements on the understanding of the different gluon distribution functions, as a clean signature for gluon saturation and to constrain saturation models further, has been explored. It is shown that dihadron correlation measurements will be one of the key methods to probe gluon saturation phenomena at a future EIC.
\end{abstract}

\section{INTRODUCTION}

It is well-known that the concept of parton distribution functions (PDFs) and their linear evolution through the Dokshitzer-Gribov-Lipatov-Altarelli-Parisi (DGLAP) formalism [1 [3] is a key ingredient for perturbative quantum chromodynamics (pQCD) and has been successfully applied to understand many hard processes in high energy hadronic collisions. Using extractions of PDFs from pQCD fits to cross section data [4], a large number of high-energy physics experiments have established that parton distributions, especially the gluon distribution, grow rapidly as the partonic longitudinal momentum fraction, $x$, gets smaller. The high-energy evolution of gluon density at fixed momentum transfer, $Q^{2}$, is described by the so-called Balitsky-Fadin-KuraevLipatov (BFKL) evolution equation [5]. The BFKL equation is a linear evolution equation and it governs the evolution of the gluon distribution with respect to $x$. Its solution exhibits a rapid increase as $x$ gets smaller. However, the gluon density cannot grow arbitrarily large, since this would violate the unitarity limit for forward scattering amplitudes or the Froissart bound for total cross sections at very high energies. Recent experimental data at rather small $x$ have provided us some intriguing evidence for the existence of a novel QCD regime, namely the saturation regime, which cannot be fully described by linear QCD evolution approaches $[\underline{6}-8]$.

The idea of saturation physics can be briefly described as follows: when the gluon density at low $x$ becomes so large that different gluon clouds with fixed transverse size $\sim 1 / Q^{2}$ start to overlap with each other,

\footnotetext{
* lzheng@bnl.gov

† elke@bnl.gov

$\ddagger$ jhlee@bnl.gov

$\S$ xiaobowen@phy.ccnu.edu.cn
}

the QCD evolution dynamics essentially becomes nonlinear [9, 10]. It is conceivable that gluons can recombine in a dense medium, thereby taming further rapid growth of the gluon density. The non-linear extension of the BFKL equation is given by the Balitsky-Kovchegov equation [11]. This non-linear dynamical effect can be enhanced with a nuclear target, where the interaction develops over a longitudinal distance of the order of the nuclear size or larger. In this case the nucleons located at the same impact factor cannot be distinguished from each other. Gluons from different nucleons can amplify the total transverse gluon density by a factor of $A^{1 / 3}$ for a nucleus with mass number $A$. Typically, a characteristic scale $Q_{s}(x, A)$ can be introduced to describe the transition to the saturation region. For $Q^{2}>Q_{s}^{2}$, the target hadron is usually treated as a dilute system, whereas $Q^{2}<Q_{s}^{2}$ corresponds to the case with a highly dense saturated hadron with a large parton density. Therefore, one can define a boundary with $Q^{2}=Q_{s}^{2}(x)$ in the $x-Q^{2}$ plane to describe the transition from the non-linear saturation regime to the linear dilute regime. The main physical ingredient of the saturation formalism is to incorporate the unitarity constraint for high-energy scattering amplitudes through the inclusion of non-linear recombination in the quantum evolution of hadronic wave functions.

Although it is not conclusive that such a saturated regime has been discovered at presently running highenergy experimental facilities, it has certainly received a great amount of theoretical support with the development of the Color Glass Condensate (CGC) effective field theory [12, 13]. To achieve an accurate description of experimental data it is essential to have theoretical calculations beyond leading order, especially as the higherorder corrections are known to be sizeable. This analysis accounts for gluon radiation in the calculation of the dihadron cross section through the inclusion of Sudakov factors. It is one of the most important corrections com- 
puted on one-loop level. This is vital for the comparison between the theory calculation and the future ElectronIon Collider (EIC) [14, 15] data on dihadron correlations.

It has been a challenging task to constrain to what extent saturation is present in available experimental data [16]. Azimuthal dihadron correlations are considered to be a very compelling measurement to tell whether the partonic system under study has reached the saturation regime or not [17. The azimuthal angle $(\Delta \phi)$ distribution of correlated high- $p_{T}$ hadron pairs uncovers the underlying jet properties on a statistical basis. The near-side peak $(\Delta \phi=0)$ of this $\Delta \phi$ distribution is dominated by the fragmentation from the leading jet, while the away-side peak $(\Delta \phi=\pi)$ is expected to be dominated by back-to-back jets produced in the hard $2 \rightarrow 2$ scattering. At sufficiently high parton densities, when saturation effects dominate, incoming gluons normally carry a typical transverse momentum at a scale of $Q_{s}>Q$, which significantly increases the transverse momentum imbalance of the back-to-back jets. As a result, saturated gluons from the target tend to smear the back-toback picture and suppress the away-side peak in the $\Delta \phi$ distribution.

The observed suppressions in dihadron correlation measurements at forward rapidities performed in $d+\mathrm{Au}$ $\sqrt{s}=200 \mathrm{GeV}$ collisions at RHIC [18 22] are perhaps the most suggestive evidence of the onset of the saturation regime in present data. Rather significant suppression of the away-side correlation is observed, when one compares the data for central $d+\mathrm{Au}$ collisions to peripheral $d+\mathrm{Au}$ collisions at forward rapidities. The qualitative feature of this suppression was first predicted by Marquet 23] based on saturation physics/CGC calculations. The strength of back-to-back correlations and the depletion of the away-side peak measured in these experiments can be quantitatively described in the saturation formalism [24 26].

At an EIC, we can access dihadron correlations in deep inelastic scattering (DIS) data from $e+\mathrm{A}$ and $e+p$ collisions, which can provide a clean and well-controlled signature of saturation physics complementary to the current $d+\mathrm{Au}$ or $p+\mathrm{A}$ measurements. They also provide the opportunity to study a fundamental gluon distribution that cannot be accessed today. It has been shown in the recent theoretical development of small $x$ physics that there are two different unintegrated gluon distributions (UGDs); namely the Weizsäcker-Williams (WW) gluon distribution and the dipole gluon distribution, which are involved in the calculation of various observables [27]. Since all other gluon distributions appearing in various processes can be constructed from these two UGDs in the large $N_{c}$ limit of QCD, they can be considered as the universal and fundamental building blocks for all UGDs. Furthermore, the WW gluon distribution can be interpreted as the gluon density in the light cone gauge, while the dipole gluon distribution has no such probabilistic interpretation. In addition, we want to emphasize that the WW gluon distribution only appears in few physical processes exclusively, and currently there is very little knowledge about its behavior. Fortunately, the WW gluon distribution is the only UGD involved in the DIS dijet process [29], which provides us a unique and clean means to measure the WW gluon distribution.

In this paper, based on the most recent theoretical developments in saturation physics, we perform a detailed study of the feasibility, expected precision, and physics impact of dihadron correlation measurements on gluon dynamics in the small $x$ region at a future highluminosity, high-energy EIC. We will demonstrate that, at a future EIC such as eRHIC at BNL or MEIC at JLab (see Sec.5 of Ref. [15] and references therein for more details), it is feasible to perform the discussed dihadron correlation measurement even with a moderate integrated luminosity of $\mathcal{L}=1 \mathrm{fb}^{-1}$. We present results for two lepton-nucleus beam energy configurations, $10 \mathrm{GeV} \times$ $100 \mathrm{GeV}$ and $20 \mathrm{GeV} \times 100 \mathrm{GeV}$, and compare the results for proton and gold beams. We use pseudo-data generated by the Monte Carlo generator PYTHIA 28] integrated with nuclear PDFs, geometry and energy loss to obtain a non-saturation baseline. The framework of Ref. [29] is used to obtain numerical predictions and to study the size of the suppression of dihadron correlations in a saturation formalism. Table $\square$ shows the definitions of the kinematic variables used in this study.

TABLE I: Kinematic variables

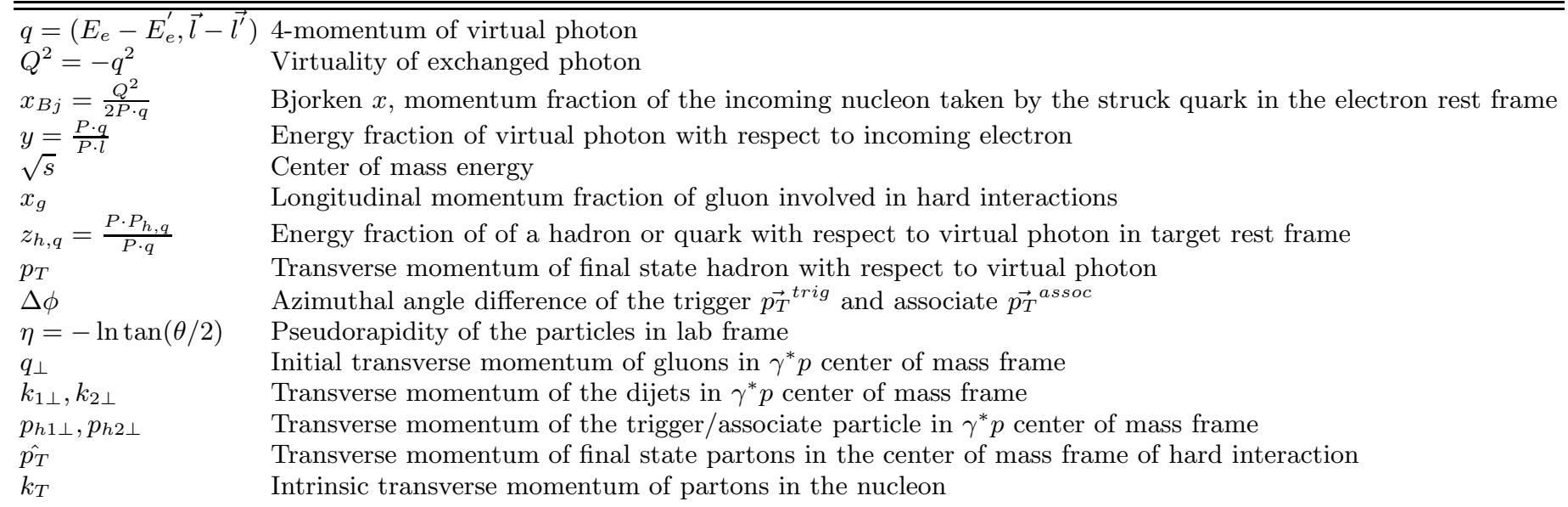


The rest of this article is organized as follows: in Sec. II, we discuss the theoretical framework used for the prediction of saturation effects in the dihadron correlation measurement. A brief comparison of dihadron correlations in $e+\mathrm{A}$ versus $p+\mathrm{A}$ is provided in Sec. III. In Sec. IV] we give an overview of the planned EIC project and present simulation results for dihadron correlations at an EIC. Finally, we summarize and conclude in Sec. V

\section{DIHADRON CORRELATIONS IN THE SATURATION FORMALISM}

According to the effective small- $x k_{t}$ factorization established in Ref. [29], which is briefly summarized above, the back-to-back correlation limit of the dihadron production cross section can be used to directly probe the WW gluon distribution $x G^{(1)}\left(x, q_{\perp}\right)$. As a comparison, the hadron production in semi-inclusive deep inelastic scattering (SIDIS), as shown in Ref. 31], is related to the so-called dipole gluon distributions $x G^{(2)}\left(x, q_{\perp}\right)$.

The coincidence probability $C(\Delta \phi)=\frac{N_{\text {pair }}(\Delta \phi)}{N_{\text {trig }}}$ is a commonly exploited observable in dihadron correlation studies, in which $N_{\text {pair }}(\Delta \phi)$ is the yield of the correlated trigger and associate particle pairs, while $N_{\text {trig }}$ is the trigger particle yield. This correlation function $C(\Delta \phi)$ depends on the azimuthal angle difference $\Delta \phi$ between the trigger and associate particles. In terms of theoretical calculation, the correlation function is defined as

$$
C(\Delta \phi)=\frac{1}{\frac{d \sigma_{\text {SIDIS }}^{\gamma^{*}+A h_{1}+X}}{d z_{h 1}}} \frac{d \sigma_{\text {tot }}^{\gamma^{*}+A \rightarrow h_{1}+h_{2}+X}}{d z_{h 1} d z_{h 2} d \Delta \phi}
$$

Let us consider a process of a virtual photon scattering on a dense nuclear target producing two final state back-to-back $q \bar{q}$ jets: $\gamma^{*}+A \rightarrow q\left(k_{1}\right)+\bar{q}\left(k_{2}\right)+X$, in which $k_{1}$ and $k_{2}$ are the four momenta of the two outgoing quarks. This process is the dominant one in the low- $x$ region, since the gluon distribution is much larger than the quark distributions inside a hadron at high energy. The back-to-back correlation limit indicates that the transverse momentum imbalance is much smaller than each individual momentum: $q_{\perp}=\left|k_{1 \perp}+k_{2 \perp}\right| \ll P_{\perp}$, with $P_{\perp}$ defined as $\left(k_{1 \perp}-k_{2 \perp}\right) / 2$. At leading order (LO), the dihadron total cross section, which includes both the longitudinal and transverse contributions, can be written as follows [29]:

$$
\begin{gathered}
\frac{d \sigma_{\text {tot }}^{\gamma^{*}+A \rightarrow h_{1}+h_{2}+X}}{d z_{h 1} d z_{h 2} d^{2} p_{h 1 \perp} d^{2} p_{h 2 \perp}}=C \int_{z_{h 1}}^{1-z_{h 2}} d z_{q} \frac{z_{q}\left(1-z_{q}\right)}{z_{h 2}^{2} z_{h 1}^{2}} d^{2} p_{1 \perp} d^{2} p_{2 \perp} \mathcal{F}\left(x_{g}, q_{\perp}\right) \mathcal{H}_{\mathrm{tot}}\left(z_{q}, k_{1 \perp}, k_{2 \perp}\right) \\
\times \sum_{q} e_{q}^{2} D_{q}\left(\frac{z_{h 1}}{z_{q}}, p_{1 \perp}\right) D_{\bar{q}}\left(\frac{z_{h 2}}{1-z_{q}}, p_{2 \perp}\right)
\end{gathered}
$$

where $C=\frac{S_{\perp} N_{c} \alpha_{e m}}{2 \pi^{2}}$ gives the normalization factor, with $S_{\perp}$ being the transverse area of the target, $z_{q}$ is the longitudinal momentum fraction of the produced quark with respect to the incoming virtual photon, $\mathcal{H}_{\text {tot }}$ is the combined hard factor, $k_{1 \perp}$ and $k_{2 \perp}$ are the transverse momenta of the two quarks, while $p_{h 1 \perp}$ and $p_{h 2 \perp}$ are the transverse momenta of the two corresponding produced hadrons respectively. $\mathcal{F}\left(x_{g}, q_{\perp}\right)$ comes from the relevant WW gluon distribution $x G^{(1)}\left(x_{g}, q_{\perp}\right)$ evaluated with the gauge links for a large nucleus at small $x$ by using the McLerran-Venugopalan model [12],

$\mathcal{F}\left(x_{g}, q_{\perp}\right)=\frac{1}{2 \pi^{2}} \int d^{2} r_{\perp} e^{-i q_{\perp} r_{\perp}} \frac{1}{r_{\perp}^{2}}\left[1-\exp \left(-\frac{1}{4} r_{\perp}^{2} Q_{s}^{2}\right)\right]$

in which $x_{g}=\frac{z_{q} p_{h 1}^{2}}{z_{h 1}^{2} s}+\frac{\left(1-z_{q}\right) p_{h 2 \perp}^{2}}{z_{h 2}^{2} s}+\frac{Q^{2}}{s}$ is the longitudinal momentum fraction of the small- $x$ gluon with respect to the target hadron and $Q_{s}$ is the gluon saturation scale. $D_{q}\left(\frac{z_{h}}{z_{q}}, p_{\perp}\right)$ represents the transverse momentum dependent fragmentation functions, where $p_{\perp}$ shows the additional transverse momentum introduced by frag- mentation processes. There can be more sophisticated model description of the WW gluon distribution, which involves a numerical solution to the BK type evolution for the WW gluon distribution [34, 35]. But studying the impact of these PDFs is beyond the scope of this work presented here.

In principle, the so-called linearly polarised gluon distribution [32, 33] also contributes to the dihadron correlation and can be systematically taken into account. This part of the contribution comes from an averaged quantum interference between a scattering amplitude and a complex conjugate amplitude with active gluons linearly polarized in two orthogonal directions in the azimuthal plane. Numerical calculation shows that this contribution is negligible for dihadron back-to-back correlations. Also, this type of contribution vanishes when the dihadron correlation function is averaged over the azimuthal angle of the trigger particle.

As to the single-inclusive-hadron production cross section, which enters the denominator of the definition of the correlation function $C(\Delta \phi)$, it can be calculated from the 


$$
\begin{gathered}
\frac{d \sigma_{\mathrm{SIDIS}}^{\gamma^{*}+A \rightarrow h_{1}+X}}{d z_{h 1} d^{2} p_{h 1 \perp}}=C \int_{z_{h 1}}^{1} d z_{q} \int d^{2} q_{\perp} F_{x_{g}}\left(q_{\perp}\right) H_{\mathrm{SIDIS}}\left(k_{\perp}, q_{\perp}, Q\right) \\
\times \sum_{q} e_{q}^{2} \frac{z_{q}}{z_{h 1}^{2}} D_{q}\left(\frac{z_{h 1}}{z_{q}}, p_{\perp}\right)
\end{gathered}
$$

where $H_{\text {SIDIS }}$ is the $q_{\perp}$ dependent hard factor for SIDIS, which includes both the longitudinal and transverse photon contribution. Here $F_{x_{g}}\left(q_{\perp}\right)$, which is related to $x G^{(2)}\left(x_{g}, q_{\perp}\right)$, is the Fourier transform of the dipole cross section:

$$
\begin{aligned}
F_{x_{g}}\left(q_{\perp}\right) & =\int \frac{d^{2} r}{2 \pi^{2}} e^{i q_{\perp} \cdot r_{\perp}} \frac{1}{N_{c}} \operatorname{Tr}\left\langle U\left(r_{\perp}\right) U^{\dagger}(0)\right\rangle_{\rho} \\
& \simeq \frac{1}{\pi Q_{s A}^{2}} \exp \left[-\frac{q_{\perp}^{2}}{Q_{s A}^{2}}\right] .
\end{aligned}
$$

It has been suggested in Refs [34, 35] that both dipole and WW gluon distributions have similar geometric scaling behavior. Therefore, one can parameterize these gluon distributions following the Golec-Biernat Wüsthoff (GBW) [36] model calculation, in which $Q_{s A}^{2}(x)=$ $c(b) A^{1 / 3} Q_{s 0}^{2}\left(x / x_{0}\right)^{-\lambda}$, with $Q_{s 0}=1 \mathrm{GeV}, x_{0}=3.04 \times$ $10^{-4}$ and $\lambda=0.288$. The gluon saturation momentum is related to $Q_{s A}^{2}(x)$ by $Q_{s}^{2}(x)=\frac{2 N_{c}^{2}}{N_{c}^{2}-1} Q_{s A}^{2}(x)$. $c(b)=c(0) \sqrt{1-b^{2} / R^{2}}$ gives the nuclear profile depen- dence with a radius $R$, where $b$ is the impact parameter. As it is not an easy task to determine the exact impact parameter in $e+\mathrm{A}$ collisions, a median number $c(b)=0.8$ is used for the estimation, which is supposed to average the nucleus geometry effectively. The parametrized DSS fragmentation function $[37], D\left(z, p_{\perp}\right)=D(z) \frac{1}{\pi\left\langle p_{\perp}^{2}\right\rangle} e^{\frac{-p^{2}}{\left\langle p_{\perp}^{2}\right\rangle}}$ with $\left\langle p_{\perp}^{2}\right\rangle=0.2 \mathrm{GeV}^{2}$, is used to compute the hadron production.

By utilizing Eq. (2) and Eq. (4), one can straightforwardly calculate the coincidence probability. The theoretical prediction at the Born level for the suppression of the away-side of the dihadron correlation measurement is shown by the solid curves in Fig. 1

All the above results are estimated based on the LO Born level contribution. At the EIC energy scale the one-loop contribution [39], which is also known as the so-called Sudakov factor, can be important as well. To include the Sudakov factor contribution at leading double logarithm level, one can rewrite the relevant WW distribution as follows [40]:

$$
\mathcal{F}\left(x_{g}, q_{\perp}\right)=\frac{1}{2 \pi^{2}} \int d^{2} r_{\perp} e^{-i q_{\perp} r_{\perp}} \frac{1}{r_{\perp}^{2}}\left[1-\exp \left(-\frac{1}{4} r_{\perp}^{2} Q_{s}^{2}\right)\right] \exp \left[-\frac{\alpha_{s} N_{c}}{4 \pi} \ln ^{2} \frac{K^{2} r_{\perp}^{2}}{c_{0}^{2}}\right]
$$

where $K^{2}$ represents the hard momentum scale in twoparticle production processes. It can be chosen as $K^{2}=$ $P_{\perp}^{2}$ or $K^{2}=Q^{2}$, depending on which one is larger, and $c_{0}=2 e^{-\gamma_{E}}$ with the Euler constant $\gamma_{E}$. It is known that the single logarithmic terms as well as the next-toleading order (NLO) contribution of the Sudakov factor also have sizeable contributions compared to the above leading double logarithmic contribution. Therefore, the numerical value of $\alpha_{s}$ in the Sudakov factor used in this calculation may be different from what one normally expects according to a QCD running coupling constant calculation.

One needs to pay attention to the applicability of this calculation. As the GBW model is not sufficient to describe the UGDs in the region where $q_{\perp}$ is much larger than $Q_{s}$, we should limit this calculation to the saturation region $\left(x_{g}<0.01\right)$ to ensure the GBW model can be applied. Additionally, to ensure that the power corrections to the two-particle production are negligible, one needs the magnitude of the jet transverse momenta $P_{\perp}$ to be much larger than $Q_{s}$.

The current calculations are performed for $Q^{2}$ of the same order as $P_{\perp}^{2}$. For pair production, the Sudakov factor is usually due to a scale difference between $P_{\perp}$ and the dijet momentum imbalance $q_{\perp}$. Because we have required that $P_{\perp} \gg q_{\perp}$ as discussed above, it is necessary to include the Sudakov contribution. As for the trigger hadron inclusive cross section, the Sudakov factor is not important, since the trigger hadron $p_{T}$ is of the same order as $Q$ and $P_{\perp}$. An illustration of this Sudakov effect with $\alpha_{s}=0.35$ can be found in Fig. 1 labeled by the dashed lines. It is worthwhile to point out that the Sudakov effect in a nuclear environment is still not very well known. In the current small $x$ scenario as shown in Eq. (6), it is convoluted with the gluon distribution function. The theoretical calculation indicates that the Sudakov factor has no nuclear $A$ dependence at LO. As shown in Fig. 1, the away-side suppression of 


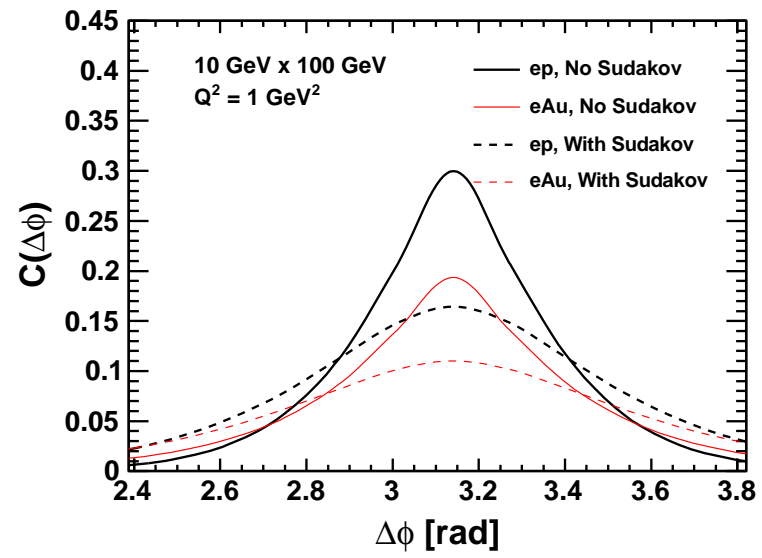

FIG. 1. [color online] $\pi^{0}$-correlation curves calculated in the saturation formalism at $10 \mathrm{GeV} \times 100 \mathrm{GeV}$ for $e+p$ (thick line) and $e+\mathrm{Au}$ (thin line) with (dashed curve) and without (solid curve) the Sudakov factor. The kinematics chosen are $y=0.7, Q^{2}=1 \mathrm{GeV}^{2}, z_{h 1}=z_{h 2}=0.3, p_{h 1 \perp}>$ $2 \mathrm{GeV} / c, 1 \mathrm{GeV} / c<p_{h 2 \perp}<p_{h 1 \perp}$.

the dihadron correlation is due to the combination of the Sudakov suppression and saturation effects. It is conceivable that the suppression due to saturation effects shall become more and more dominant when the ion beam species are changed from proton to gold, while the $\mathrm{Su}-$ dakov effect remains more or less the same.

\section{CONNECTIONS TO $P+$ A DIHADRON CORRELATIONS}

Compared to existing $p+\mathrm{A}$ or $d+\mathrm{Au}$ dihadron correlation data, there are several advantages to measuring dihadron correlations in $e+\mathrm{A}$ collisions. One valuable feature is that one can make use of the scattered electron to reconstruct kinematic information event by event. Measuring the scattered electron allows us to model-independently determine the required kinematic variables $x$ and $Q^{2}$, which is essential for probing the underlying gluon dynamics precisely. Another advantage comes from the point-like structure of electrons. Since electrons have no substructure and they couple to virtual photons rather weakly, the probability to have multiple emission in $e+\mathrm{A}$ is very small compared to $p+\mathrm{A}$. This kind of multiple emission or interaction introduces a significant amount of uncorrelated two-particle production, which is known as the "pedestal" effect in RHIC $d+\mathrm{Au}$ collisions. To understand the so-called pedestal effect, one needs to take into account the double parton scattering, which includes two independent and uncorrelated hard scatterings. In contrast, as explained above, the pedestal contribution should be negligible and under control in the $e+\mathrm{A}$ dihadron correlation measurement.

In addition, as is shown in Fig 2, the kinematic coverage of the planned eRHIC realization of the EIC is very similar to the measured RHIC $d+\mathrm{Au}$ data and extends to the desired small- $x$ region. Therefore, it will be a more precise and definite measurement compared to what we already know from the RHIC $d+\mathrm{Au}$ data. Here, the RHIC kinematics lines are calculated with the assumption that the major fraction of the parton energy is mainly taken by the hadrons, which is not always true.

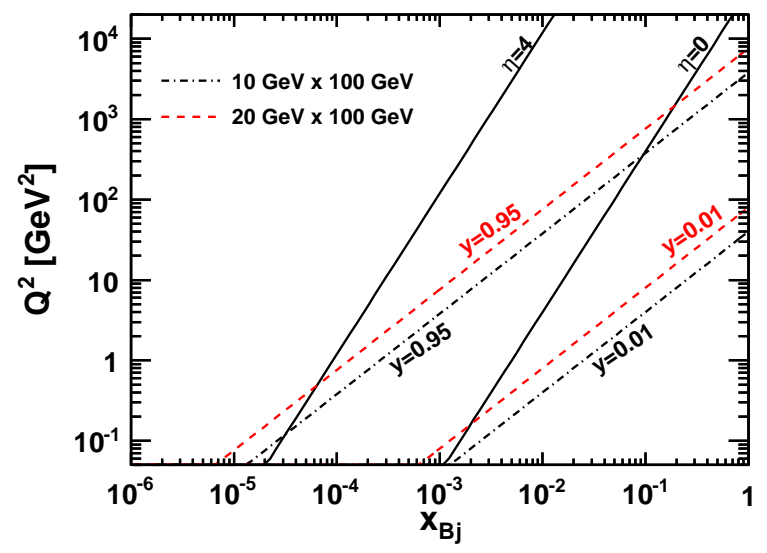

FIG. 2. [color online] The eRHIC kinematics coverage compared to $p+\mathrm{A}$ at RHIC. The dash-dotted and dashed lines show the eRHIC kinematics for the beam energies of $10 \mathrm{GeV}$ $\times 100 \mathrm{GeV}$ and $20 \mathrm{GeV} \times 100 \mathrm{GeV}$, respectively. The solid lines represent the RHIC coverage at $\sqrt{s}=200 \mathrm{GeV}$ for $\eta=0$ and $\eta=4$, where $\eta=-\ln \tan (\theta / 2)$ is the pseudorapidity of the particles.

The measurement of dihadron correlations in $e+\mathrm{A}$ collisions is interesting by itself. It provides us with a golden opportunity to directly measure the saturated WW gluon distribution. Through detailed calculations, Ref. [29] summarizes the involvement of these two basic gluon distributions in different observables. It is interesting to note that the dipole gluon distribution function is involved in most known processes, especially inclusive DIS measurements, which provides us with a lot of the essential information of the dipole scattering amplitude. On the other hand, the WW gluon distribution contributes to only a few of these processes, thus very little knowledge about the WW distribution exists from the current experimental data. In addition, unlike the dijet process in $p+\mathrm{A}$, which receives contribution from both the dipole and WW gluon distribution, the WW gluon distribution is the only UGD that contributes to $e+\mathrm{A}$ Dijet production. Considering that the WW gluon distribution can be physically interpreted as the number density of gluons inside a nuclear wave function, while the dipole gluon distribution does not have such interpretation, it is important and fundamental to acquire direct information on the WW gluon distribution through dihadron correlation measurements at an EIC. 


\section{ELECTRON-ION COLLIDER AND SIMULATIONS}

\section{A. The Electron-Ion Collider and its Detector}

Two independent designs for an EIC are being developed in the United States: eRHIC, at Brookhaven National Laboratory (BNL); and MEIC/ELIC at Thomas Jefferson National Laboratory (JLab). The following studies will focus on the eRHIC version of an EIC and the new model detector at eRHIC. The eRHIC design at BNL reuses the available infrastructure and facilities of RHIC's high-energy polarized proton/ion beam. A new electron beam, based on Energy Recovery LINAC (ERL) technology, is to be built inside the current RHIC tunnel. At eRHIC, the collision luminosity is expected to be in the order of $10^{33-34} \mathrm{~cm}^{-2} \mathrm{~s}^{-1}$. The full range of proton/ion beam energies will be accessible from the beginning of operations, while the electron beam energy will start with $10-15 \mathrm{GeV}$ and later be increased to 20 $\mathrm{GeV}$.

The tracking system of the baseline eRHIC detector will consist of a TPC, GEM and silicon detectors spanning a range of $-4<\eta<4$ in pseudorapidity. The endcap and barrel region on the detector will be equipped with electromagnetic calorimeters covering $-4.5<\eta<$ 4.5. Hadronic calorimeter will be used mostly for jet physics at full energy in the forward (hadron beam going direction) and backward (electron beam going direction) rapidities spanning $2<|\eta|<4$.5. Projected momentum and energy resolutions of these devices are better than a few percent, which extends the capability of this detector to a large variety of physics topics.

The present study is based on the planned lepton and nucleon beam energy of $10 \mathrm{GeV} \times 100 \mathrm{GeV}$ and 20 $\mathrm{GeV} \times 100 \mathrm{GeV}$. The kinematics are constrained to the main region of interest for dihadron correlation studies, $1 \mathrm{GeV}^{2}<Q^{2}<20 \mathrm{GeV}^{2}$ and $0.01<y<0.95$. Backward electromagnetic calorimetry and the TPC are utilized for reconstruction of the event kinematics (based on the scattered electron). As shown in Fig. 3. abundant high- $p_{T}$ particles will be generated in the specified kinematic region to make correlated hadron pairs even with a limited luminosity of $1 \mathrm{fb}^{-1}$. Among those generated high- $p_{T}$ particles, Fig. 4 suggests that gluon dijet processes dominate in the production of particles with a transverse momentum greater than $2 \mathrm{GeV} / c$ and charged pions are the major component for the final state particles.

\section{B. Monte Carlo Set Up}

The simulation part of this study is based on the PYTHIA-6.4 Monte Carlo program, with the PDF input from the LHAPDF library [42] and JETSET used for fragmentation processes. If one defines $\hat{p_{T}}$ as the transverse momentum of final state partons in the cen- ter of mass system of the hard interaction, the factorization scale $\mu^{2}$ of $2 \rightarrow 2$ processes can be expressed as $\mu^{2}={\hat{p_{T}}}^{2}+\frac{1}{2} Q^{2}$.

In PYTHIA, depending on the wave function components for the incoming virtual photon, various subprocesses are divided into three major classes: the direct processes, the VMD processes and the anomalous processes [43], as illustrated in Fig. 5] The direct photon interacts as a point-like particle with the partons of the nucleon, while the VMD and anomalous components interact through their hadronic structure. The VMD component is characterized by non-perturbative fluctuations of the photon into a $q \bar{q}$ pair existing long enough to evolve into a hadronic state before the subsequent interaction with the nucleon [44]. This process can be described in the VMD model, where the hadronic state is treated as a vector meson (e.g. $\left.\rho^{0}, \omega, \phi\right)$ with the same quantum numbers as the photon. These VMD states can undergo all the soft/hard interactions with the nucleon allowed in hadronic physics. The large-scale, perturbatively fluctuated photons can be added as the anomalous photon part in a Generalized VMD (GVMD) model. Same pQCD $2 \rightarrow 2$ process can be developed over the VMD or anomalous state of the virtual photons on the target nucleon, with the difference that parameterized photon PDFs are used for anomalous photons whereas for the hard VMD components those vector meson PDFs are involved. Hard VMD and anomalous process are usually referred to as "resolved" process. Resolved photon processes play a significant part in the production of hard high- $p_{T}$ processes at $Q^{2} \approx 0$. The following hard subprocesses are grouped in the resolved processes category: $q q \rightarrow q q, q \bar{q} \rightarrow q \bar{q}, q \bar{q} \rightarrow g g, q g \rightarrow q g, g g \rightarrow q \bar{q}, g g \rightarrow g g$. In the high- $Q^{2}$ region, direct processes become dominant, Fig. 6] shows the major subprocesses in that category: LO DIS, Photon-Gluon Fusion (PGF) and QCD Compton (QCDC). As the PGF process is directly sensitive to the gluon distribution, it is extremely important for DIS dijet productions. The final conditional yield can be expressed as a superposition from different processes:

$$
C(\Delta \phi)=\Sigma_{i} w_{i} w_{i}^{s} C(\Delta \phi)_{i},
$$

with $w_{i}$ being the statistical weight of every subprocess $i$ involved in the measurement and $w_{i}^{s}$ is the suppression factor for the subprocess $i$ from saturation. For the quark channels unaffected by saturation $w_{i}^{s}=1$, while for the gluon channels, suppression of $C(\Delta \phi)$ at away-side is expected with $w_{i}^{s}<1$.

To simulate $e+p$ events the CTEQ6M [41] PDF in the $\overline{\mathrm{MS}}$ scheme is used. For the $e+\mathrm{A}$ event sample, the NLO EPS09 parton distribution functions [45] and hard parton energy loss based on the medium geometry have been applied to account for nuclear effects in the simulation. Modifications of nuclear PDF have the form

$$
f_{i}^{A}\left(x, Q^{2}\right)=R_{i}^{A}\left(x, Q^{2}\right) f_{i}^{p}\left(x, Q^{2}\right),
$$

where $R_{i}^{A}$ is the nuclear modification factor multiplied on top of the free proton PDF $f_{i}^{p}\left(x, Q^{2}\right)$ for a parton of 

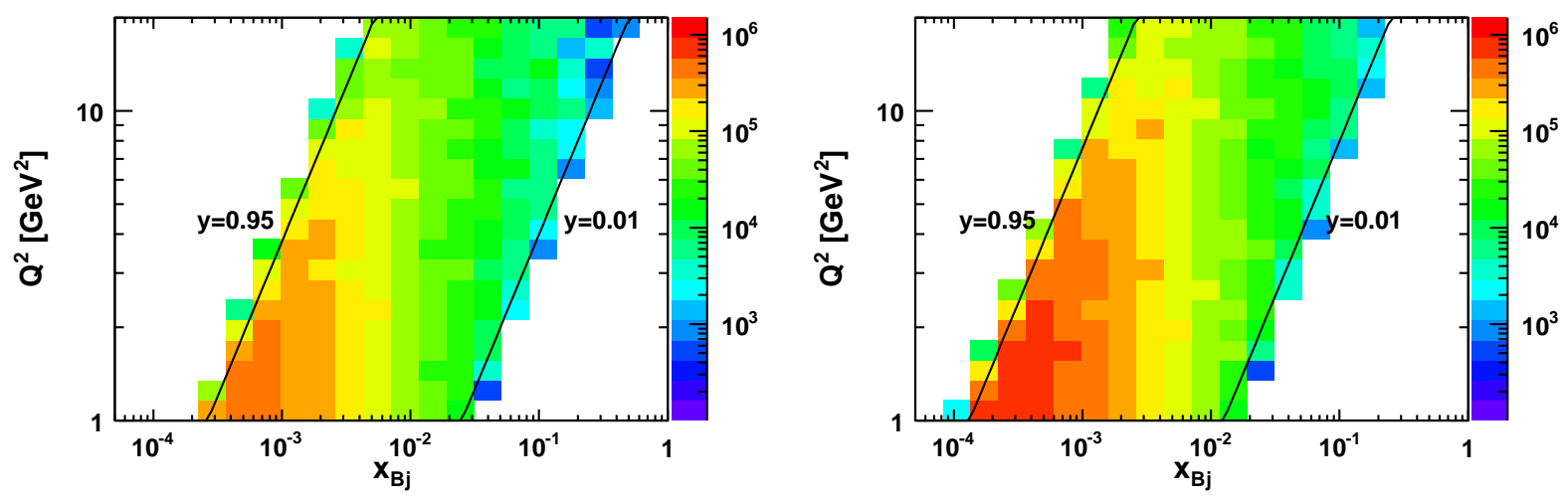

FIG. 3. [color online] Expected yields of charged particle pairs at transverse momentum $p_{T}>1 \mathrm{GeV} / c$ in bins of $\left(Q^{2}, x_{B j}\right)$ for an integrated luminosity of $1 \mathrm{fb}^{-1}$ for $e+p 10 \mathrm{GeV} \times 100 \mathrm{GeV}$ (Left) and $20 \mathrm{GeV} \times 100 \mathrm{GeV}$ (Right) in the kinematic range of $1 \mathrm{GeV}^{2}<Q^{2}<20 \mathrm{GeV}^{2}$, and $0.01<y<0.95$.
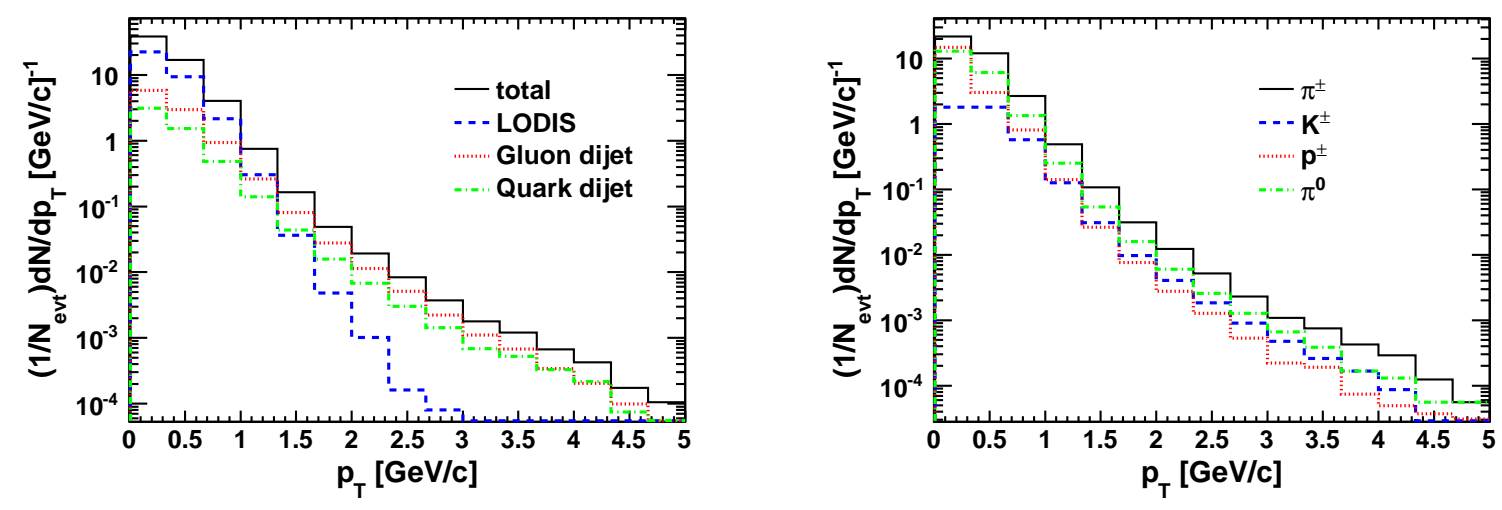

FIG. 4. [color online] Particle $p_{T}$ distributions for $e+p 10 \mathrm{GeV} \times 100 \mathrm{GeV}$ collisions with $1 \mathrm{GeV}^{2}<Q^{2}<20 \mathrm{GeV}^{2}, 0.01<y<$ 0.95. Left: charged particle production from LO DIS, gluon dijets (PGF and resolved gluon channel) and quark dijets (QCDC and resolved quark channel). Right: $\pi^{ \pm}, K^{ \pm}, p^{ \pm}$and $\pi^{0}$ production for all processes.

flavor $i$, with $f_{i}^{p}\left(x, Q^{2}\right)$ being the CTEQ6M PDF set. Assuming isospin symmetry for protons and neutrons, the up/down quark distribution can be averaged according to the corresponding mass number $A$ and charge number $Z$. The hard parton energy loss in the nuclear medium is included following the parton quenching model (PQM) formalism based on Ref [46]. In the energy loss picture, the medium effect is characterized by the so-called transport coefficient, defined as the average medium-induced squared transverse momentum per unit path length for a hard parton:

$$
\hat{q}=\left\langle k_{\perp}^{2}\right\rangle_{\text {medium }} / \lambda,
$$

where $\lambda$ is the mean free path and $k_{\perp}$ represents the medium-introduced transverse momentum to the hard parton. The characteristic energy loss scale is set by

$$
\omega_{c}=\frac{1}{2} \hat{q} L^{2}
$$

$L$ is the medium path length the parton traverses through. It is determined by the impact parameter at which the hard scattering occurs with respect to the geometry of the nucleus described by a Wood-Saxon distribution. Using the above parameters, one can define the energy loss probability distribution as follows

$$
P\left(\Delta E ; R, \omega_{c}\right)=p_{0}(R) \delta(\Delta E)+p\left(\Delta E ; R, \omega_{c}\right),
$$

where $p_{0}$ is the probability that the parton experiences no medium induced radiation.

In the phenomenological studies of dihadron correlations, triggering on a hadron with high- $p_{T}$ on average selects the most energetic hadron in events with back-toback jets. Correlated hadron pairs reflect two important features of QCD dynamics of the hard scattering process. First, an associated hadron at the near-side allows one to probe the in-medium QCD evolution of an energetic parton, which can be viewed as the final state effect with 


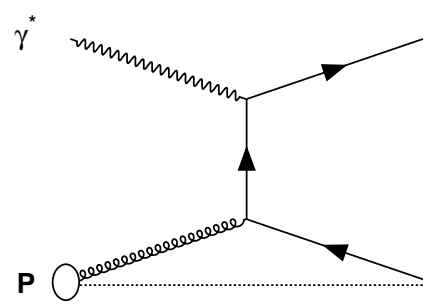

(a)

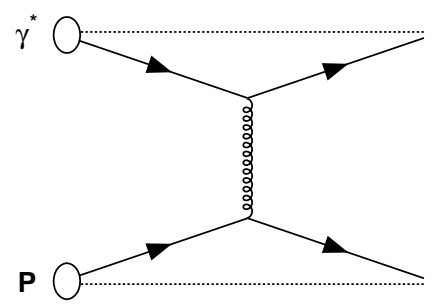

(b)

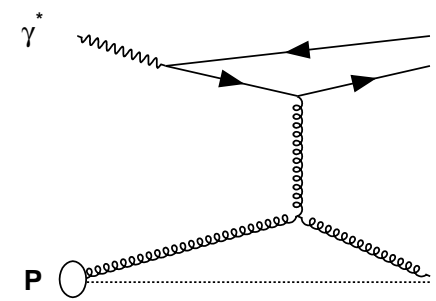

(c)

FIG. 5. Feynman diagrams for different PYTHIA subprocesses contributing to the hard interaction: (a) direct, (b) VMD, (c) anomalous. The dotted lines indicate the presence of a spectator. Bubbles stand for a hadron or hadronic structure.

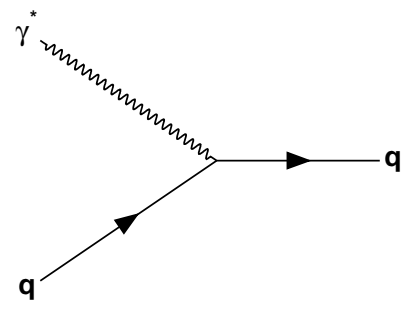

(a)

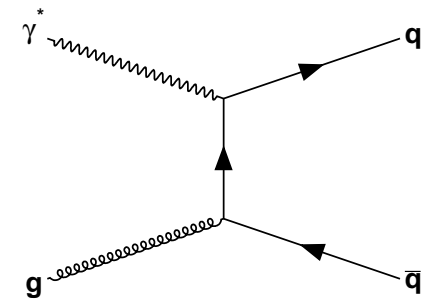

(b)

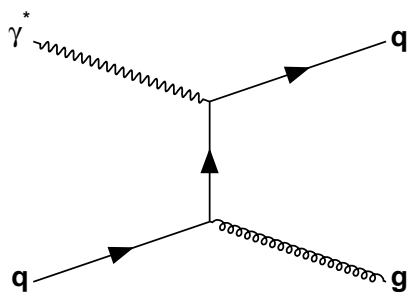

(c)

FIG. 6. Feynman diagrams for the hard processes based on point-like photons: (a) $\mathcal{O}\left(\alpha_{s}^{0}\right)$ LO DIS, (b) Photon-Gluon Fusion (PGF) and (c) QCD Compton scattering (QCDC).

the nuclear medium. Second, an associated hadron at the away-side, in addition to the primary hard scattering, is sensitive to the initial transverse momentum that the incoming parton carries.

For pQCD calculations in the collinear factorization framework, the PDFs and fragmentation functions do not contain any transverse momentum dependence. Therefore, the transverse momentum of hadrons produced in the final state is given by $p_{T}=z \hat{p_{T}}$, where $\hat{p_{T}}$ and $p_{T}$ are the transverse momentum of the parton and hadron respectively. $z$ represents the momentum fraction of a hadron with respect to its mother parton. This relation should be revised if the transverse momentum is allowed in both the PDFs and fragmentation functions.

Transverse motion of partons inside hadrons can be effectively included by assuming that the intrinsic $k_{T}$ follows a Gaussian distribution. Similarly, the transverse momentum enhancement $p_{T}^{\text {frag }}$ with respect to the jet direction during hadronization can also be approximated by a Gaussian distribution. The intrinsic $k_{T}$ and fragmentation $p_{T}^{\text {frag }}$ now both contribute to the transverse momentum of final state hadrons, which can be written as $p_{T}=z\left(k_{T}+\hat{p_{T}}\right)+p_{T}^{\text {frag }}$. We follow the common practice to set the Gaussian width to $0.4 \mathrm{GeV}$ for both intrinsic $k_{T}$ and $p_{T}^{\text {frag }}$ distributions in the simulations.

Besides all the above effects, additional soft gluon radiations, normally characterized as a parton shower can also modify the final transverse momentum, thereby impacting the dihadron correlations. In perturbative QCD calculations the parton shower are computed in terms of Sudakov form factors.

Fig. 7 shows an illustration of all the possible effects available in the Monte Carlo in the simulation of the azimuthal correlation function. The open circles illustrate the dihadron correlation with only intrinsic $k_{T}$ in the initial parton distribution. It is understandable that the correlation function is strongly peaked at $\Delta \phi=0, \pi$ for this setting. Now the other effects are turned on one-byone according to the order of their occurrences in physical processes. When the initial state (IS) parton shower is added into the simulation, as shown by the open diamonds, the away-side correlation is significantly reduced since it is very sensitive to the momentum imbalance of the dijet system, while the near-side correlation is almost unmodified. Next, we turn on the final state (FS) parton shower for the scattered parton before the fragmentation process occurs. We find that both the near-side and away-side peaks are broadened, as illustrated by the empty triangles, due to soft radiation and particle decay in the fragmentation. Lastly, we add transverse momentum dependence into the fragmentation function, labeled as $p_{T}^{\text {frag }}$, and obtain the crossings, which indicate further broadening of both peaks.

For our model of $e+\mathrm{A}$ implemented in PYTHIA, the effects due to energy loss in the cold nuclear medium are expected to be weak, because fast moving partons are likely to fragment outside the nucleus in the considered kinematic regions. Considering that the nuclear PDF also has little impact on the $p_{T}$ imbalance of dijets, it 


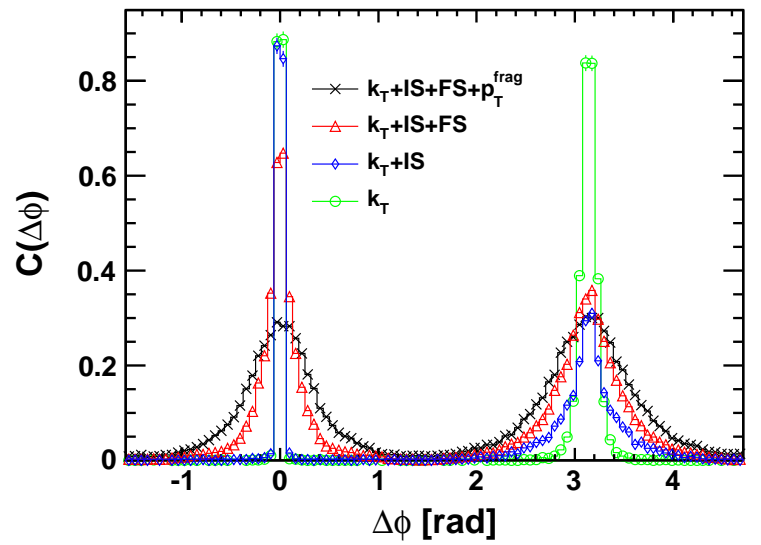

FIG. 7. [color online] Comparison of dihadron correlation due to different physical inputs, such as intrinsic $k_{T}$, initial state parton shower (IS), final state parton shower plus resonance decay (FS) and $p_{T}$ broadening in fragmentation processes. The $e+p$ data are for charged hadrons with a beam energy of $20 \mathrm{GeV} \times 100 \mathrm{GeV}$ with $1.0 \mathrm{GeV}^{2}<Q^{2}<1.5 \mathrm{GeV}^{2}, 0.65<$ $y<0.75, p_{T}^{\text {trig }}>2 \mathrm{GeV} / c, 1 \mathrm{GeV} / c<p_{T}^{\text {assoc }}<p_{T}^{\text {trig }}, 0.2<$ $z_{h}^{\text {trig }}, z_{h}^{\text {assoc }}<0.4$.

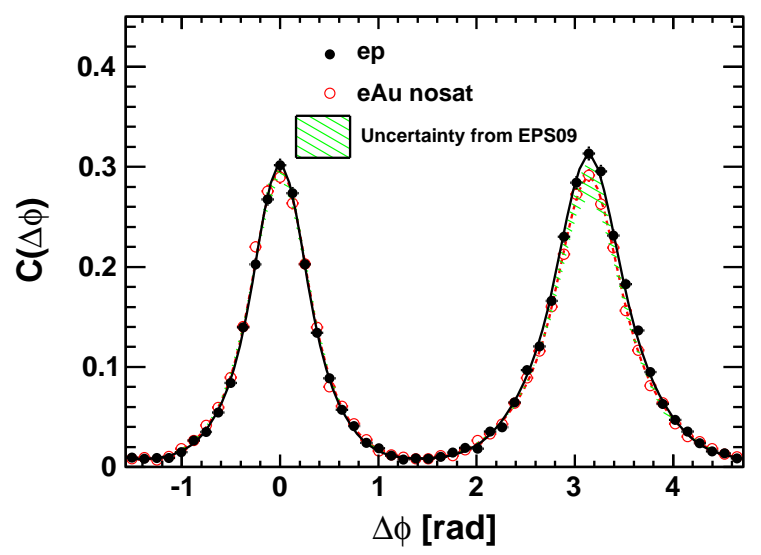

FIG. 8. [color online] Simulated data points for particle correlations for charged hadrons in $e+p$ and $e+\mathrm{Au}$ collisions with beam energies of $20 \mathrm{GeV} \times 100 \mathrm{GeV}$ and $1.0 \mathrm{GeV}^{2}<$ $Q^{2}<1.5 \mathrm{GeV}^{2}, 0.65<y<0.75, p_{T}^{\text {trig }}>2 \mathrm{GeV} / c, 1 \mathrm{GeV} / c<$ $p_{T}^{\text {assoc }}<p_{T}^{\text {trig }}, 0.25<z_{h}^{\text {trig }}, z_{h}^{\text {assoc }}<0.35$. Lines are the fit for $e+p$ or $e+\mathrm{A}$ points. The shaded band shows the uncertainty due to the EPS09 nuclear PDFs.

comes as no surprise to see very little change from $e+p$ to $e+\mathrm{A}$ in the simulation, as shown in Fig. 8. Table II is a reference for different effects on the relative root mean square (RMS) deviation of the near/away side azimuthal correlation function, from which we can clearly draw the conclusion that initial-state parton showers dominate the away-side peak of the correlation function, while the
TABLE II. Relative Root Mean Square (RMS) for the $\Delta \phi$ distribution from $e+p$ collisions including different effects influencing the width of the near and away side peak compared to the baseline RMS with all the effects included (bottom row).

\begin{tabular}{lcc}
\hline \hline & Near-side $\Delta \phi$ RMS & Away-side $\Delta \phi$ RMS \\
\hline$k_{T}$ & 0.21 & 0.25 \\
$k_{T}+$ IS & 0.30 & 0.72 \\
$k_{T}+$ IS + FS & 0.65 & 0.81 \\
$k_{T}+$ IS + FS $+p_{T}^{\text {frag }}$ & 1.00 & 1.00 \\
\hline \hline
\end{tabular}

near-side peak is mainly controlled by final-state effects such as final-state parton showers, fragmentation $p_{T}$ and possible resonance decays in the fragmentaion.

As the saturation physics discussed above is mainly about the gluon dynamics, in order to be able to consistently compare with the theoretical dihadron cross section in Sec. III, we need to include gluon dijet channels from PGF and gluon-initiated resolved process in the comparison. However, as the measured observable in the real experiment is a mixture of different process, as illustrated in Eq. (7), we have to know how significant the signal from gluon saturation manifests itself in a mixed event sample. From the saturation-based predictions, a sizeable suppression of the away-side peak from $e+p$ to $e+\mathrm{A}$ is expected.

In the meanwhile, it is crucial to point out that parton showers suppress the away-side peak of the dihadron correlation function just like saturation does. However, currently it is still unclear how the parton shower effect is modified in the nuclear medium, without which it is hard to draw any definite conclusions about the saturation effects, as parton showers and saturation effects are always entangled. Nevertheless, thanks to the large kinematic coverage of eRHIC, one can explore the nuclear dependence of parton showers outside the saturation region by measuring dihadron correlations for different nuclei in the high $Q^{2}$ regime. This kinematic regime has a significant phase space for parton showers for this observable. More importantly, the measurement of dihadron correlations gives the opportunity to use the near-side peak of the correlation function as a reference to study the nuclear medium effects on parton showers as the saturation effects only manifest themselves in the away-side peak, as shown in Fig. 7

In the saturation formalism, the parton shower contribution is effectively cast into the Sudakov factor for the DIS dijet process at small $x$. To illustrate this point, Fig. 9 shows the correlation function simulated with and without parton showers, compared to the corresponding theoretical predictions with and without Sudakov effects. The filled circles represent the PYTHIA simulation for $e+p$ without parton showers, and they agree very well with the solid line from the theoretical prediction including saturation effects, but excluding Sudakov effects. The comparison (empty circles and dashed line) between simulated PYTHIA $e+p$ data including parton showers and the theoretical predictions with saturation plus Sudakov 


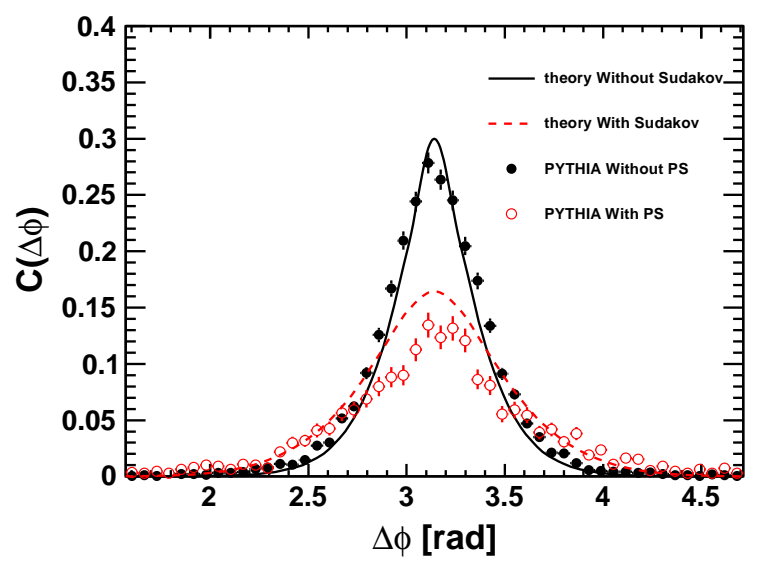

FIG. 9. [color online] $\pi^{0} \Delta \phi$ correlation comparing PYTHIA and theoretical saturation calculations for $e+p 10 \mathrm{GeV} \times$ $100 \mathrm{GeV}$ for events from PGF and resolved gluon channel subprocesses at $1.0 \mathrm{GeV}^{2}<Q^{2}<2.0 \mathrm{GeV}^{2}, \quad 0.65<y<$ $0.75, p_{T}^{\text {trig }}>2 \mathrm{GeV} / c, 1 \mathrm{GeV} / c<p_{T}^{\text {assoc }}<p_{T}^{\text {trig }}, 0.25<$ $z_{h}^{\text {trig }}, z_{h}^{\text {assoc }}<0.35$. The solid and dashed curves show theoretical predictions including saturation effects for $e+p$ without and with Sudakov factor, respectively. The filled and empty circles illustrate PYTHIA simulations for $e+p$ without and with parton showers.

effects is also good, especially considering the model uncertainties. Thus, the agreement in $e+p$ collisions enables one to estimate the nuclear medium effects on parton showers in the theoretical predictions for saturation including Sudakov effects.

Since the saturation effect decouples from hadronization, it does not depend on which specific particle type being detected. Although the theoretical prediction is made for $\pi^{0}$, the suppression factor from $e+p$ to $e+\mathrm{A}$ still holds for other different final state particles. In the next section, the significance for the suppression of gluon saturation will be shown for the charged hadrons $C(\Delta \phi)$ observable with limited statistics and expected background estimation.

\section{Monte Carlo Results and Uncertainties}

In order to guarantee the validity of perturbative calculations and avoid the kinematic regime of quasi-real photo-production, a cut of $Q^{2}>1 \mathrm{GeV}^{2}$ is generally made. On the other hand, probing the saturation dynamics requires to probe the dense region, which means that one needs to go to low $x$ and low-to-moderate $Q^{2}$ in the pursuit of saturation effects at a certain center of mass energy. A cut in transverse momentum of the charged hadron pairs is usually performed to pick particles from hard interactions. A cut on $z_{h}$ is also imposed to reject particles from the target remnants. Thus, a typical cut to select dihadron pairs from hard parton

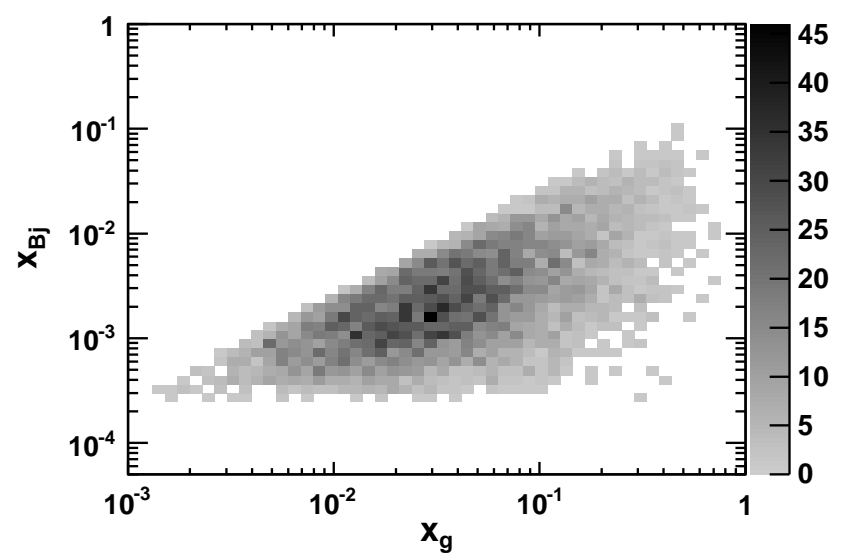

FIG. 10. The correlation of $x_{B j}$ vs $x_{g}$ for the PGF process for $e+p$ at $10 \mathrm{GeV} \times 100 \mathrm{GeV}$ with $0.01<y<0.95,1 \mathrm{GeV}^{2}<$ $Q^{2}<20 \mathrm{GeV}^{2}$. A relatively broad correlation between these two variables is observed.

scatterings is: $p_{T}^{\text {trig }}>2 \mathrm{GeV} / c, 1 \mathrm{GeV} / c<p_{T}^{\text {assoc }}<$ $p_{T}^{\text {trig }}, 0.2<z_{h}^{\text {trig }}, z_{h}^{\text {assoc }}<0.4$. To further explore the transition behavior in and out of the saturation region, we use three $Q^{2}$ bins: $1 \mathrm{GeV}^{2}<Q^{2}<2 \mathrm{GeV}^{2}, 3 \mathrm{GeV}^{2}<$ $Q^{2}<5 \mathrm{GeV}^{2}$ and $9 \mathrm{GeV}^{2}<Q^{2}<20 \mathrm{GeV}^{2}$; and two $y$ bins: $0.25<y<0.35$ and $0.6<y<0.8$. To study saturation physics using varying heavy ion beams, we focus on the $1 \mathrm{GeV}^{2}<Q^{2}<2 \mathrm{GeV}^{2}, 0.6<y<0.8$ bin, while the $9 \mathrm{GeV}^{2}<Q^{2}<20 \mathrm{GeV}^{2}$ or $0.25<y<0.35$ bins serve as a reference for the behaviour without saturation effects. To pin down the nuclear dependence of parton showers, we compare the correlation between $e+p$ and $e+\mathrm{A}$ collisions for bins without saturation effects. Because the saturation scale $Q_{s}$ varies with the gluon momentum fraction $x_{g}$, it is important to have access to $x_{g}$. Fig. 10] shows how, by utilizing $x_{B j}$, one can effectively constrain the underlying $x_{g}$ distribution. Although this is only a broad correlation, it is demonstrated in Fig. [11 that the typical $x_{g}$ at a given $x_{B j}$ is constrained to a certain magnitude and can be used to separate the saturation region from the non-saturated region.

Fig. 12 shows the $\eta$ distribution of the trigger particle and the correlated particle in the aforementioned kinematic bins at $10 \mathrm{GeV} \times 100 \mathrm{GeV}$ and $20 \mathrm{GeV} \times 100$ $\mathrm{GeV}$. Clearly, with a charged particle acceptance spanning $-4.5<\eta<4.5$, both trigger and associate particles in our kinematics binning scheme can be fully accepted by the detector.

In Fig. 13 we compare the strength of the coincidence probability based on a theoretical saturation model prediction for the away-side for $e+p$ and $e+A u$. The filled circles in Fig. 13 are simulated with PYTHIA for $e+p$ collisions, including detector smearing and acceptance effects. The projected $e+\mathrm{Au}$ saturated correlation function (open circles) is obtained by multiplying 


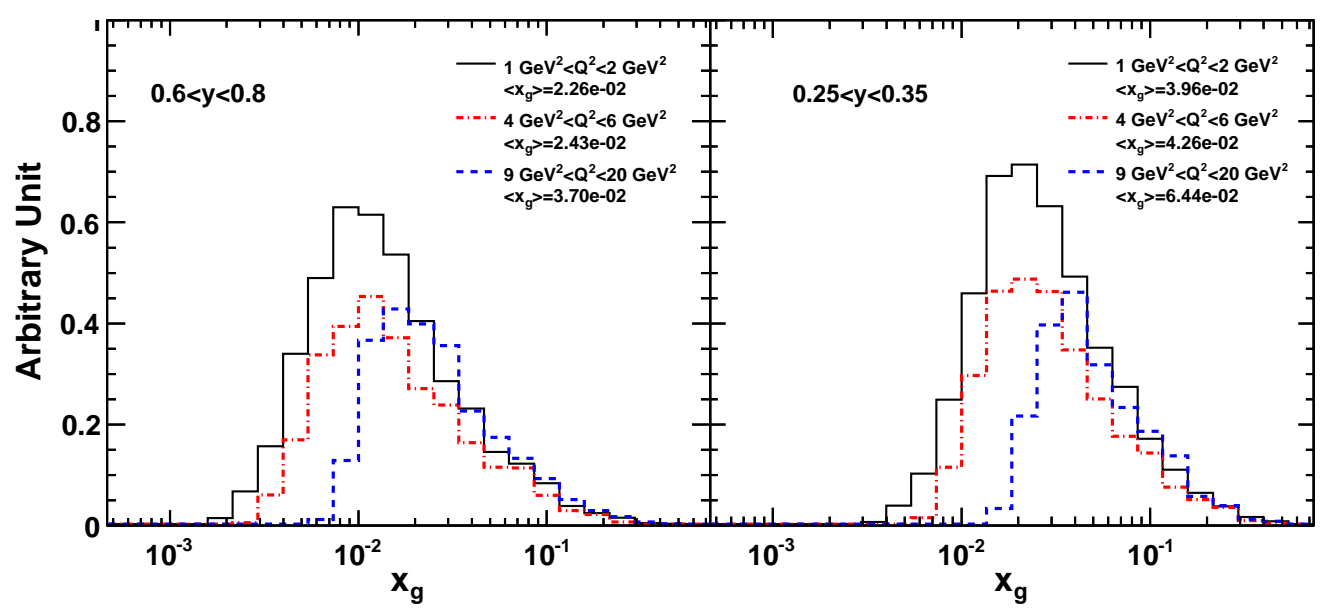

FIG. 11. [color online] $x_{g}$ distributions in various kinematics bins probed by the correlated hadron pairs in the PGF process for $e+p 10 \mathrm{GeV} \times 100 \mathrm{GeV}$.

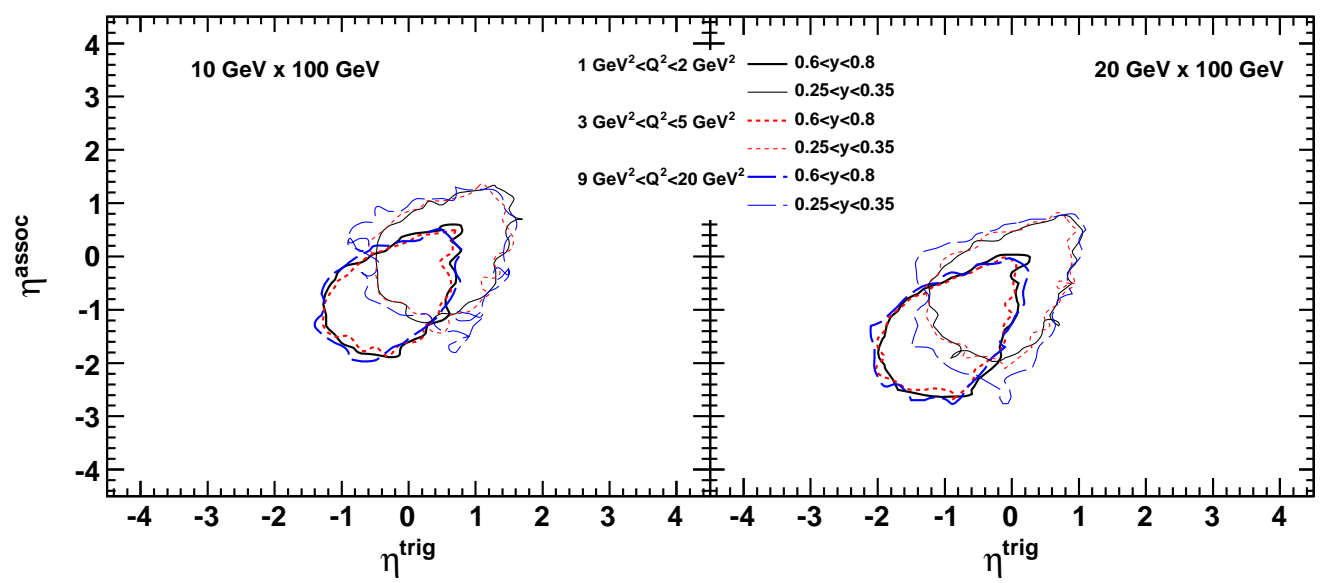

FIG. 12. [color online] The contours show the $\eta$ regions covered by the correlated dihadron pairs: for $10 \mathrm{GeV} \times 100 \mathrm{GeV}$ and $20 \mathrm{GeV} \times 100 \mathrm{GeV}$. Thick lines mark out the region for $0.6<y<0.8$ while thin lines for $0.25<y<0.35$.

the $e+p$ histogram with the suppression factor $w_{i}^{s}=$ $C(\Delta \phi)_{e A u} / C(\Delta \phi)_{e p}$ including Sudakov effects extracted from Fig. 1. This suppression factor can only be applied to dijet channels involving gluons; namely PGF and resolved $q g \rightarrow q g, g g \rightarrow g g, g g \rightarrow q \bar{q}$ subprocesses. The other quark initiated subprocesses have been simulated with PYTHIA using the non-saturated $e+\mathrm{A}$ model including nuclear PDFs and final-state energy loss. The uncertainties represent the statistical precision from an integrated luminosity of $1 \mathrm{fb}^{-1}$. The solid (dashed) lines in Fig. 13 represent fits to the simulated data points with (without) detector effects included in the simulation.

Since to date there is no exact knowledge of the saturation scale, the uncertainty in the suppression factor is estimated by varying the saturation scale by a factor of 0.5 and 2 . The resulting uncertainty bands are depicted in Fig. 13. The suppression of the away-side peak remains significant even with this additional uncertainty compared to the $e+\mathrm{Au}$ curve shown in Fig. 8 accounting for nuclear effects in the parton distribution functions, energy loss effects and the resonance decay. In summary, the suppression effects on dihadron correlations due to saturation can be clearly discriminated from effects based on classical nuclear medium modifications with a well-designed EIC machine. 


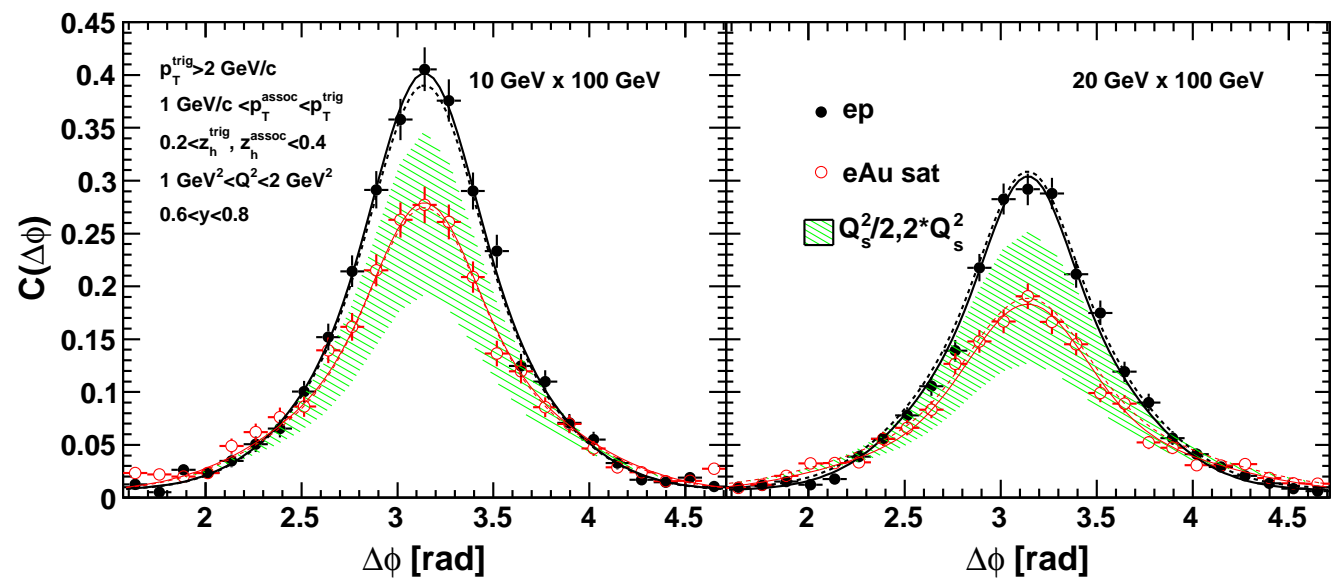

FIG. 13. [color online] The correlation function at $1 \mathrm{GeV}^{2}<Q^{2}<2 \mathrm{GeV}^{2}, 0.6<y<0.8$ for an integrated luminosity of $1 f b^{-1}$. The $e+p$ result comes from PYTHIA simulations. The $e+\mathrm{Au}$ results are a combination of simulations from a saturation-based model plus modified PYTHIA simulations. The suppression factor uncertainty was estimated by varying $Q_{s}^{2}$ by a factor of 0.5 and 2. Sudakov resummation has also been incorporated for $e+\mathrm{Au}$. The solid lines represent a fit for the simulated pseudo-data including detector effects; the dashed line excludes detector effects.

\section{SUMMARY}

Through detailed analysis, we have shown the capability of a proposed EIC to perform dihadron correlation measurements. It is proven that the acceptance of the dedicated detector is wide enough to collect all the trigger particles as well as the associated particles used in our studies. Moreover, the onset of the projected saturation region is well covered by the eRHIC energy regime. It will clearly be possible to do a high precision measurement of the correlation function for dihadron production with different nuclear beams at the proposed EIC.

In this study we also describe how this dihadron correlation function is calculated in a saturation/CGC formalism, and provide predictions for this measurement with or without saturation effects taken into consideration. It is straightforward to see that a strong suppression of the away-side peak of the correlation function is expected from saturation effects, and detector effects are negligible on this observable. Suppression effects due to leadingtwist shadowing are significantly smaller. Therefore, the observation of such a suppression in the dihadron correlation function measured at an EIC will be a strong experimental evidence for the existence of gluon saturation.

Dihadron measurements at an EIC are also vital and intriguing in that they will directly measure for the first time the behavior of the Weizsäcker-Williams gluon distribution, about which we still know very little, and which we can hardly extract from other measurements. The knowledge of how parton showers behave in a nu- clear medium is indispensable in obtaining a valid conclusion for the above discussions. With the Sudakov resummation performed in the saturation formalism, this nuclear modification of parton showers in DIS dijet process is found to be very small at leading order. Nevertheless, there might be some nuclear dependence in the $\mathrm{Su}$ dakov factor at higher orders or in the non-perturbative part 47]. An EIC will also permit unique measurements that will give a definite answer to this question. With the wide kinematic reach and different nuclear beams, an EIC is capable of measuring the A dependence of parton showers. This would require quantitative measurements of the modification of the near-side peak of the correlation functions in a nucleus environment and in kinematic regions where parton showers dominate.

In conclusion, the proposed high-luminosity, highenergy Electron-Ion Collider, together with the designed detector, can provide an ideal apparatus to study gluon saturation with high precision through the measurement of the dihadron correlation function.

\section{ACKNOWLEDGMENTS}

This work was supported in part by the NSFC (11375071), the National Basic Research Program of China (2013CB837803), and the Basic Research Program of CCNU (CCNU13F026). E.C.A. and J.H.L. acknowledge support by the U.S. Department of Energy under contract number DE-AC02-98CH10886. 
[1] Y. L. Dokshitzer, Sov. Phys. JETP 46, 641 (1977) [Zh. Eksp. Teor. Fiz. 73, 1216 (1977)].

[2] V. N. Gribov and L. N. Lipatov, Sov. J. Nucl. Phys. 15, 438 (1972) [Yad. Fiz. 15, 781 (1972)].

[3] G. Altarelli and G. Parisi, Nucl. Phys. B 126, 298 (1977).

[4] F. D. Aaron et al. [H1 and ZEUS Collaboration], JHEP 1001, 109 (2010) [arXiv:0911.0884 [hep-ex]].

[5] I. I. Balitsky and L. N. Lipatov, Sov. J. Nucl. Phys. 28, 822 (1978) [Yad. Fiz. 28, 1597 (1978)]; E. A. Kuraev, L. N. Lipatov and V. S. Fadin, Sov. Phys. JETP 45, 199 (1977) [Zh. Eksp. Teor. Fiz. 72, 377 (1977)].

[6] A. M. Stasto, K. J. Golec-Biernat and J. Kwiecinski, Phys. Rev. Lett. 86, 596 (2001) [hep-ph/0007192].

[7] N. Armesto, C. A. Salgado and U. A. Wiedemann, Phys. Rev. Lett. 94, 022002 (2005) [hep-ph/0407018].

[8] F. Gelis, E. Iancu, J. Jalilian-Marian and R. Venugopalan, Ann. Rev. Nucl. Part. Sci. 60 (2010) 463 [arXiv:1002.0333 [hep-ph]].

[9] L. V. Gribov, E. M. Levin and M. G. Ryskin, Phys. Rept. 100, 1 (1983).

[10] A. H. Mueller and J. -W. Qiu, Nucl. Phys. B 268, 427 (1986).

[11] I. Balitsky, Nucl. Phys. B463, 99-160 (1996); Y. V. Kovchegov, Phys. Rev. D60, 034008 (1999).

[12] L. D. McLerran and R. Venugopalan, Phys. Rev. D 49, 2233 (1994) [hep-ph/9309289].

[13] E. Iancu, A. Leonidov and L. McLerran, hep-ph/0202270.

[14] D. Boer, M. Diehl, R. Milner, R. Venugopalan, W. Vogelsang, D. Kaplan, H. Montgomery and S. Vigdor et al., arXiv:1108.1713 [nucl-th].

[15] A. Accardi, J. L. Albacete, M. Anselmino, N. Armesto, E. C. Aschenauer, A. Bacchetta, D. Boer and W. Brooks et al., arXiv:1212.1701 [nucl-ex].

[16] J. L. Albacete and C. Marquet, Nucl. Phys. A 854, 154 (2011) [arXiv:1009.3215 [hep-ph]].

[17] D. Kharzeev, E. Levin and L. McLerran, Nucl. Phys. A 748, 627 (2005) [hep-ph/0403271].

[18] I. Arsene et al. [BRAHMS Collaboration], Phys. Rev. Lett. 93, 242303 (2004) [nucl-ex/0403005].

[19] J. Adams et al. [STAR Collaboration], Phys. Rev. Lett. 97, 152302 (2006) [nucl-ex/0602011].

[20] A. Adare et al. [PHENIX Collaboration], Phys. Rev. Lett. 107, 172301 (2011) [arXiv:1105.5112 [nucl-ex]].

[21] E. Braidot [STAR Collaboration], Nucl. Phys. A 854, 168 (2011) [arXiv:1008.3989 [nucl-ex]].

[22] X. Li [STAR Collaboration], Nucl. Phys. A904-905 2013, 823c (2013) [arXiv:1212.1686].

[23] C. Marquet, Nucl. Phys. A 796, 41 (2007) [arXiv:0708.0231 [hep-ph]].

[24] J. L. Albacete and C. Marquet, Phys. Rev. Lett. 105, 162301 (2010) [arXiv:1005.4065 [hep-ph]].

[25] A. Stasto, B. -W. Xiao and F. Yuan, Phys. Lett. B 716, 430 (2012) [arXiv:1109.1817 [hep-ph]].

[26] T. Lappi and H. Mantysaari, Nucl. Phys. A 908, 51 (2013) [arXiv:1209.2853 [hep-ph]].

[27] F. Dominguez, B. -W. Xiao and F. Yuan, Phys. Rev.
Lett. 106, 022301 (2011) [arXiv:1009.2141 [hep-ph]].

[28] T. Sjostrand, S. Mrenna and P. Z. Skands, JHEP 0605, 026 (2006) [hep-ph/0603175].

[29] F. Dominguez, C. Marquet, B. -W. Xiao and F. Yuan, Phys. Rev. D 83, 105005 (2011) [arXiv:1101.0715 [hep$\mathrm{ph}]$.

[30] M. Anselmino, H. Avakian, D. Boer, F. Bradamante, M. Burkardt, J. P. Chen, E. Cisbani and M. Contalbrigo et al., Eur. Phys. J. A 47, 35 (2011) [arXiv:1101.4199 [hep-ex]].

[31] C. Marquet, B. -W. Xiao and F. Yuan, Phys. Lett. B 682 (2009) 207 [arXiv:0906.1454 [hep-ph]].

[32] A. Metz and J. Zhou, Phys. Rev. D 84, 051503 (2011) [arXiv:1105.1991 [hep-ph]]; D. Boer, W. J. den Dunnen, C. Pisano, M. Schlegel and W. Vogelsang, Phys. Rev. Lett. 108, 032002 (2012) [arXiv:1109.1444 [hep-ph]];

[33] F. Dominguez, J. -W. Qiu, B. -W. Xiao and F. Yuan, Phys. Rev. D 85, 045003 (2012) [arXiv:1109.6293 [hepph]]; P. Sun, B. -W. Xiao and F. Yuan, Phys. Rev. D 84, 094005 (2011) [arXiv:1109.1354 [hep-ph]]; A. Schafer and J. Zhou, Phys. Rev. D 85, 114004 (2012) [arXiv:1203.1534 [hep-ph]]; T. Liou, Nucl. Phys. A 897, 122 (2013) [arXiv:1206.6123 [hep-ph]].

[34] F. Dominguez, A. H. Mueller, S. Munier and B. -W. Xiao, Phys. Lett. B 705, 106 (2011) [arXiv:1108.1752 [hep-ph]].

[35] A. Dumitru, J. Jalilian-Marian, T. Lappi, B. Schenke and R. Venugopalan, Phys. Lett. B 706, 219 (2011) [arXiv:1108.4764 [hep-ph]].

[36] K. J. Golec-Biernat and M. Wusthoff, Phys. Rev. D 59, 014017 (1998) [hep-ph/9807513].

[37] D. de Florian, R. Sassot and M. Stratmann, Phys. Rev. D 75, 114010 (2007) [hep-ph/0703242 [hep-ph]].

[38] M. Strikman and W. Vogelsang, Phys. Rev. D 83, 034029 (2011) [arXiv:1009.6123 [hep-ph]].

[39] A. H. Mueller, B. -W. Xiao and F. Yuan, Phys. Rev. Lett. 110, 082301 (2013) [arXiv:1210.5792 [hep-ph]].

[40] A. H. Mueller, B. -W. Xiao and F. Yuan, Phys. Rev. D 88, 114010 (2013) [arXiv:1308.2993 [hep-ph]].

[41] J. Pumplin, D. R. Stump, J. Huston, H. L. Lai, P. M. Nadolsky and W. K. Tung, JHEP 0207, 012 (2002) [hep-ph/0201195].

[42] M. R. Whalley, D. Bourilkov and R. C. Group, hepph/0508110.

[43] C. Friberg and T. Sjostrand, JHEP 0009, 010 (2000) [hep-ph/0007314].

[44] T. H. Bauer, R. D. Spital, D. R. Yennie and F. M. Pipkin, Rev. Mod. Phys. 50, 261 (1978) [Erratum-ibid. 51, 407 (1979)].

[45] K. J. Eskola, H. Paukkunen and C. A. Salgado, JHEP 0904, 065 (2009) [arXiv:0902.4154 [hep-ph]].

[46] C. A. Salgado and U. A. Wiedemann, Phys. Rev. D 68, 014008 (2003) [hep-ph/0302184].

[47] Z. -B. Kang and J. -W. Qiu, Phys. Lett. B 721, 277 (2013) [arXiv:1212.6541]. 